\title{
Binary Classification Method using Invariant CSP for Hand Movements Analysis in EEG-based BCl System
}

\author{
응웬탄하 ${ }^{*}$ 박승민 ${ }^{*}$ 고광은 ${ }^{\star} \cdot$ 심귀보 ${ }^{*}$ \\ Thanh Ha Nguyen, Seung-Min Park, Kwang-Eun Ko, and Kwee-Bo Sim ${ }^{\dagger}$ \\ *중 앙대 학교 전자전기공학부 \\ + School of Electrical and Electronics Engineering, Chung-Ang University
}

\begin{abstract}
In this study, we proposed a method for electroencephalogram (EEG) classification using invariant CSP at special channels for improving the accuracy of classification. Based on the naive EEG signals from left and right hand movement experiment, the noises of contaminated data set should be eliminate and the proposed method can deal with the de-noising of data set. The considering data set are collected from the special channels for right and left hand movements around the motor cortex area. The proposed method is based on the fit of the adjusted parameter to decline the affect of invariant parts in raw signals and can increase the classification accuracy. We have run the simulation for hundreds time for each parameter and get averaged value to get the last result for comparison. The experimental results show the accuracy is improved more than the original method, the highest result reach to $89.74 \%$.
\end{abstract}

Key words : Brain-Computer Interface (BCI), Electroencephalogram (EEG), Invariant Common Spatial Pattern, Independent Component Analysis, Auditory Stimuli

\section{Introduction}

Recent research trend of brain-computer interface (BCI) system using human brain activity signals, such as electroencelophagrams (EEG) or magnetoencephalograms (MEG), translate or decode the complex-signals into commands of outer device, which correspond to user's intention. In the neuro-physiological point of view, the intention is influenced by the rhythmic activity in specific regions over the cortex. However, the brain activities, especially EEGs, have non-invasive property which noise also recorded with meaningful signal from the cortex regions. The major work of the BCI system is regarded as the detection of the difference between the intentions by selecting features and coding them to the command for controlling target systems. The non-invasive BCI systems are widely used for patients who lost limbs in accident or disorders

접수일자: 2013년 3월 8일

심사(수정)일자: 2013년 3월 28일

게재확정일자 : 2013년 3월 29일

† Corresponding author

본 논문은 한국연구재단 중견연구자지원사업(No.2012-0008726) 에서 지원하여 연구하였습니다. 연구비 지원에 감사드립니다.

This is an Open-Access article distributed under the terms of the Creative Commons Attribution Non-Commercial License (http://creativecommons.org/licenses/by-nc/3.0) which permits unrestricted non-commercial use, distribution, and reproduction in any medium, provided the original work is properly cited. which are mostly acquired. Generally, the EEGs are recorded by the user's attended responses from visual/audio stimuli. In [1-3] the EEGs were recorded during the imagination of hand, foot and tongue movement with various stimuli for inducing the target responses. The stimuli for inducing the user's attention are mostly based on visual form, for example, visually evoked $\mathrm{po}^{-}$ tential (VEP), steady-state visually evoked potential (SSVEP), and so on [4]. The visual cue-based EEGs measurement are well-known as contaminating artifacts and noise, not only insisted by unconscious muscle movements or sensitive errors of electrodes, but also caused by unwanted brain activation during the given mental tasks. In order to overcome these, some researches have applied auditory stimuli instead of visual stimuli in designing BCI systems [5-7]. The auditory cue for attention was supposed that could reduce the noise caused by eye movement or unwanted respond by eye gaze. In the other auditory experiment, subjects of experiments were instructed to closed eye and relaxed so that they could concentrate on hearing the cue and not be attacked by unwanted visual stimuli when recording signals [2].

In the BCI systems, it was supposed to be two kinds of sources are existing: brain source and noise sources [6]. It means that the quality and quantity of brain signals are influenced by signal-to-noise ratio (SNR) and the non-stationaries sources of the signals, such as subjects and time scales. These properties make the BCI systems need to be purified noise by ap- 
plying the robust non-stationary process over brain signals. In our previous work, we applied independent components analysis (ICA) to estimate the sources that caused EEGs, based on those components [8]. We assumed that brain signals were the sources that got bigger percentage of accounting variance value. From ICA technique, we could reduce the dimension of the signal from so many components contained noise. We reduced noise component aim to purify the signals. With a small number (around 20 25\% components were kept and 75 $\sim 80 \%$ of components are eliminated) of components, a new space were reconstructed. Even though, ICA could filter the noise sources caused by outer brain, but there were still artifacts that occurred inside brain which caused by non-stationary character. As mentioned above, the EEGs need to be purified outer noise by separating noise over brain sources. ICA is a very power technique to estimate the sources.

In order to solve inner noise sources, common spatial pattern (CSP) algorithm now is more famous in BCI system for spatial filter. The CSP is very useful when applied for binary choice with 2 classes, as in [4][6]. Even though, CSP is very powerful, still there are limitations that mitigate the accuracy classification [1][4][7]. The CSP extracted noise and non-stationary features, while we need it to concentrate on the meaningful data which brings the information associated with user's intention. These shortcomings are also caused by the strength of the CSP.

\section{Related Works}

\subsection{Independent Component Analysis (ICA)}

If $\vec{x}(t)$ is original $M \times N$ EEG signal and statistically $M \times N$ independent component $\vec{s}(t)$ is unknown, an instantaneous linear mixture matrix $A$ can be calculated by equation (1). The element $a_{i j}$ of $A$ is weight of mixing components [4].

$$
\vec{x}(t)=\overrightarrow{A s}(t)
$$

$A$ is always squared as $[M \times M]$, because the size of sources is equal to the size of observed signals. So we can express the inverse matrix $A$ to decoding matrix $W$ as shown in equation (2).

$$
\vec{s}(t)=A^{-1} \vec{x}(t)=\overrightarrow{W x}(t)
$$

From these sources, we could analyze and determine which sources we kept to more analyze and which sources we reduced as noise part. ICA also applied as blind source separation (BSS) in many fields of preprocessing signals.

\subsection{Common Spatial Patterns (CSP)}

It is said that CSP based on the variance and $\mathrm{CO}^{-}$ variance of signals, in which the variance of artifacts exceeded covariance matrix of endogenous component. There are some ways to extend CSP approaches. Some methods proposed to robust brain signals against noise and non-stationary changes in the data or overcome the limitation of heuristic when applied for multi-class [7]. The previous approaches have used the region of interest based on neuro-physiological to make prior to some channels data. A different CSP approach, namely stationary subspace analysis $\mathrm{SSA}+\mathrm{CSP}$, which extracted subspace of stationary signal before applied into CSP proposed stationary subspace analysis to purify EEG signals [6]. Another extension of CSP was applied into discriminant multi-class compare with information theoretic feature extraction [7][9][11]. As mentioned in [4], some of proposed approaches based on bias adaptation or update classifiers or the CSP filters. Covariance shift adaptively following shifts of the features. Moreover, Blankertz et al [5] used method called invariant CSP allows separating invariance by additional extra measurements, such as eye movement sub-session.

The CSPS has been widely used in BCI systems and highly successful in calculating spatial filter for detecting event-related potentials, especially for an decrease or increase of band-power values, namely event-related desynchronized and event - related synchronized (ERD/ERS) respectively [7]. Since variance of band-pass filtered signals is equal to band-power, CSP algorithm is suited to classify mental task that are characterized by ERD/ERS, which usually used analysis in motor activity for both actual and imagination. CSP is fit to analyze the differences in band power between two conditions. The aim of this algorithm was solved by maximizing the Rayleigh quotient for each condition.

As in [7], we assume that $C_{1}$ and $C_{2}$ are the average covariance matrices from class 1 and class $2 \mathrm{re}^{-}$ spectively, equation (3) illustrates for condition $i=1,2$ :

$$
R_{w}=\frac{w^{T} C_{i} w}{w^{T}\left(C_{1}+C_{2}\right) w}
$$

In order to solve this maximization of the Rayleigh quotient as maximize one class with:

$$
w^{T}\left(C_{1}+C_{2}\right) w-b=0
$$

, where $b$ is an arbitrary constant (the norm of $w$ can be chosen so as to satisfy the equation (4)).

Then, it can be solved by using Lagrange multiplier:

$$
L(\lambda, w)=w^{T} C_{1} w-\lambda\left(w^{T}\left(C_{1}+C_{2}\right) w-b\right)
$$

The filters $w$ can be defined by the derivative of $L$ 
with respect to $w$ equal 0 :

$$
\begin{aligned}
\frac{\partial L}{\partial w} & =2 w^{T} C_{1}-2 \lambda w^{T}\left(C_{1}+C_{2}\right)=0 \\
& \Leftrightarrow C_{1} w=\lambda\left(C_{1}+C_{2}\right) w \\
& \Leftrightarrow\left(C_{1}+C_{2}\right)^{-1} C_{1} w=\lambda w
\end{aligned}
$$

We can solve this problem by using eigenvalue and eigenvector. With largest eigenvalue corresponds to the spatial filters that maximizes the variance of class 1 $\left(C_{1}\right)$ while minimize the common variance $\left(C_{1}+C_{2}\right)$. The same progress for condition 2 and get the filter fitted to equation (3) and (4). It is simple generalization eigenvector with smallest eigenvalue. In this case the largest eigenvalue of this condition is the smallest $\mathrm{ei}^{-}$ genvalue of another condition.

\subsection{Invariant CSP (iCSP)}

The main purpose of iCSP is to extract features, not only discriminate between different conditions, but also be invariance in the same time. It means not only extracted filters that maximize and minimize between two different classes but simultaneously keep invariance along the projection as stable as possible. We added penalty term $P$ in equation (3) with parameter to adjust is $\xi$ and $P$ shows relationship between added dataset and main dataset. Added dataset is opened-eye EEG signals and closed-eye dataset is the main dataset to analyze with 2 sessions of auditory paradigm:

$$
R_{w}=\frac{w^{T} C_{i} w}{w^{T}\left(C_{1}+C_{2}+\xi P\right) w}
$$

\section{Simulation of EEG Signals Classification for Real Hand Movements}

\subsection{Raw Data Acquisition}

Fig. 1 shows our auditory paradigm for EEG acquisition during the hands movement experiment, there are three kinds of sounds, including: 1) right hand movement instruction (index $=20,500 \mathrm{~Hz}$ ), 2) left hand movement instruction (index $=10,2 \mathrm{KHz}$ ), and 3) ignored noise sound. All subjects had two sessions with different conditions: auditory and spatial auditory (which devide the auditory stimuli follow the spatial and this case is applied for left and right only so it means $180^{\circ}$ in space). We recorded EEG signals by using 64 chan- $^{-}$ nels EEG measurement system (Synamp2, NeuroScan Corp.) with the sampling rate equals $1000 \mathrm{~Hz}$. Since the experiment concentrated on the executive movement, so we recorded signals with limited 25 channels. In the terms of neuro-physiological, the limb movements are controlled by the motor cortex area over the head. For a specific analysis of hand movement [6], we chose 25 channels where cover the motor cortex, especially channels $C 3$ and $C 4$. It can be said that these 2 channels were analyzed for limb movements. Multiple channels around the two main channels are included to re $^{-}$ cord the target EEGs when playing hand movements.

Subjects were instructed to sit down and relaxed in a dark room, just concentrated to hear the sound and respond as instructed above. Data set from two subjects, who have averaging of age is 26.5 year-old and both are healthy subjects (one is left handed) and recorded with two different conditions so that we have four dataset. Along with the main data set we also recorded eye-open for additional data set for iCSP methods. All subjects are our laboratory graduate student, who the first time recorded EEG signals and BCI system.

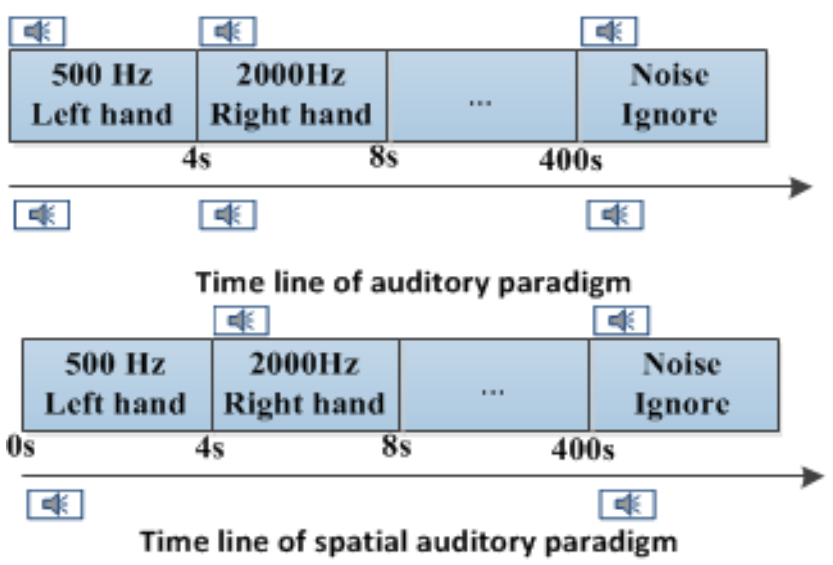

(a) Experiment time line for random auditory stimuli

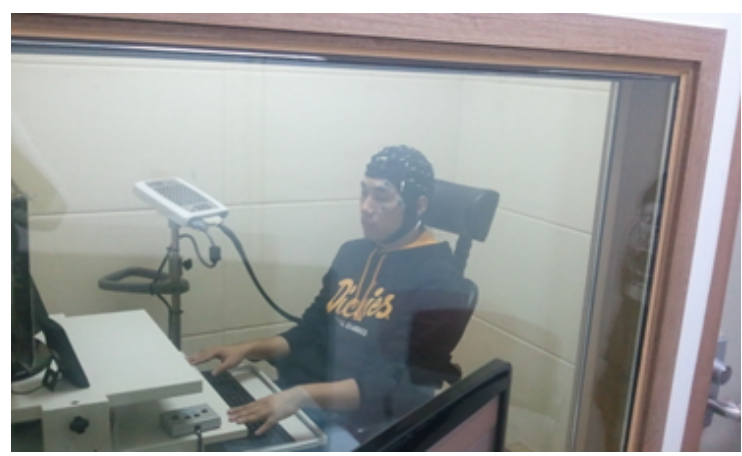

(b) Response measurement from a subject

Fig. 1. Experimental paradigm: (a) time flow line of auditory stimuli for each hand movement, (b) response measurement environment from subject for auditory and spatial auditory paradigm

In this experiment, we recorded four main dataset based on two subjects of two conditions: auditory and spatial auditory. auditory paradigm is using two side of the speaker to transmit the stimuli sound. While the spatial auditory paradigm is using only one side speaker (mono) to transmit the stimuli for subjects, so that not 
only different sound of frequency make the stimuli of right or left hand movement. It also be shown if the subject could separate the sound from right side or left side to decide right hand or left hand movement. In this time, the hand movement is real movement. When subject heard the sound based on the different frequency they decide to move right or left and then take the hand rest at the neutral pose as shown in the Fig. $1(b)$ That is also the pose when subject heard the noise sound when they ignored them. Along the four main dataset with eye closed, we also recorded the data with noise when open eye to make the additional data for iCSP method.

\subsection{Preprocessing}

Physiologically, it was shown that the limb movements activate $\mu(8 \sim 13 \mathrm{~Hz})$ and $\beta(12 \sim 25 \mathrm{~Hz})$ rhythms frequency band of EEGs [9]. It is also announced that the hand movement appeared in $\mu$ rhythms and foot movement appeared in $\beta$ rebound in the previous neuro-physiology [10][11]. Frequency domain of original signals shown the fact that the real hand movement appeared peak and high power in $\mu$ rhythm, in this case was around $8 \sim 15 \mathrm{~Hz}$ and got peak at $10 \mathrm{~Hz}$. It also shown the same fact announced in the neuro-physiological point of view [10].

In our experiment, we concentrate on $\mu$ rhythm cover the $\alpha$ band low power $(8 \sim 10 \mathrm{~Hz})$ for motor rhythms and hand movement. We used bandpass filter with 8 $13 \mathrm{~Hz}$ bandwidth for reducing the noise from odd movements. The de-noised data are continuously studied for eliminating the outer noise by using ICA algorithm. The recorded signals are whitening by bandpass filtering in the $\mu$ rhythms. Then it was consider as input for "EEGLAB" (MATLAB toolbox) to approximate the independent sources. We extracted epochs of two conditions and then removing the bad sources separately in two conditions. Based on the accounted variance, we chose the maximum covariance accumulation up to $95 \%$. In this study, the number of components were chose to be kept was around $20 \sim 30 \%$ of total 25 components. So we reduced the bad components and just kept small number of meaningful components to reconstruct and analyze. The reconstructed data is purer and reduced the noise components.

\subsection{Feature Extraction and Classification}

Data after applied preprocessing step, we analyzed for CSP to archive spatial filter for two class of hand movement. The main aim of CSP is to get the $\max ^{-}$ imum of one condition and the same time get the minimum for the rest one. By using EEGLAB, we extracted epochs of each condition. Epochs in this experiment were extracted more concentrate about the real movement when subject did and analyzing based on the different of the subject around 300ms after stimuli. In this study we based on P300 technique to analyze specific hand movement and recognition. Covariance matrices were calculated for two conditions each condition gather of 35 trials. We chose two maximum features of each condition to apply into classification step to get higher results.

As following the flowchart of the processing in this experiment in Fig. 2, we main aim to find the matrix transform $W$ to create new data space where data purer and also separately two conditions. The results were calculated by 500 times running for each value in the range of the adjusted parameter $(1 \sim 20)$ to evaluate the advance and the fit of the method and classifiers.

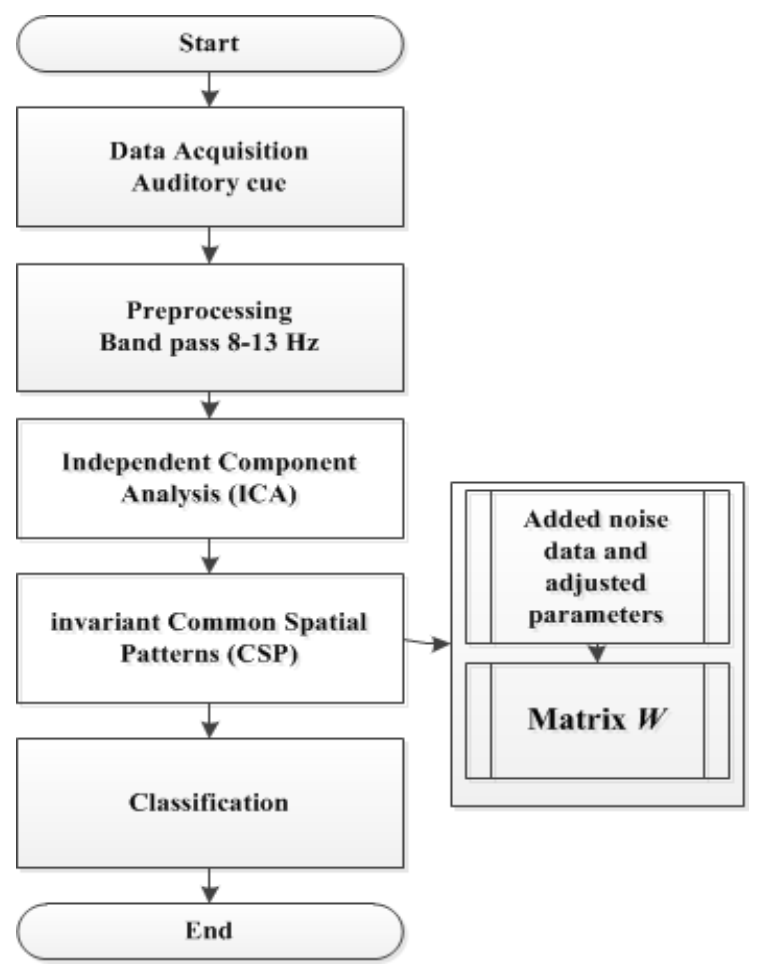

Fig. 2. Flowchart of invariant Common Spatial Pattern in BCI systems

\section{Experimental Results}

After using ICA algorithms for 25 channels, we estimated 25 components for each dataset. In each dataset we got different estimate sources so that the number of components to be kept also was different. Each data had various number of components gathered to get over $95 \%$ but it was still a very small number compared to total number in this case was 25 components. Through ICA, we kept only one over fifth of 25 components but still kept over $95 \%$ of the covariance accounted. We changed the number of trials for training to find the maximum value of accuracy. 
We applied to analyzed: $50 \%$ data for training and $50 \%$ data for testing. We used 500 times run for each value of the adjusted parameter and then calculate the averaging values to show in Fig 3. The classification results from four data sets are shown in Fig. 3 with horizontal axis corresponding the number of adjust parameter for invariant data set to de-noise the target data $\left(2^{-x}\right)$. Also, its vertical axis means the classification rates from four data sets of our two subjects playing the real hand movements. We iteratively run with hundreds times to ignore the errors in classification step. In this paper, we find out the original data still get invariant which could increase result by adding more noise data. In this case, the noises occurred when the eyes are nictating. The experimental results are compared with previous works [8] and shown improvement of the result up to $18,9 \%$ (dataset $3 \rightarrow$ proposed method: $72.9 \%$ and previous method: $54 \%$ ) for each data set (1 and 3 ). We get the result for ICA method only respectively: $58.6 \%$ and $54 \%$. So, the combination of ICA and iCSP could improve the classification accuracy, but the Fig. 1 also announced the adjust parameter got an important role to improve the classification result. The difference between each dataset go for different adjust parameter because of the fitness of dataset, e. g. each subject has different range of data to separate it.

\section{iCSP for different parameter}

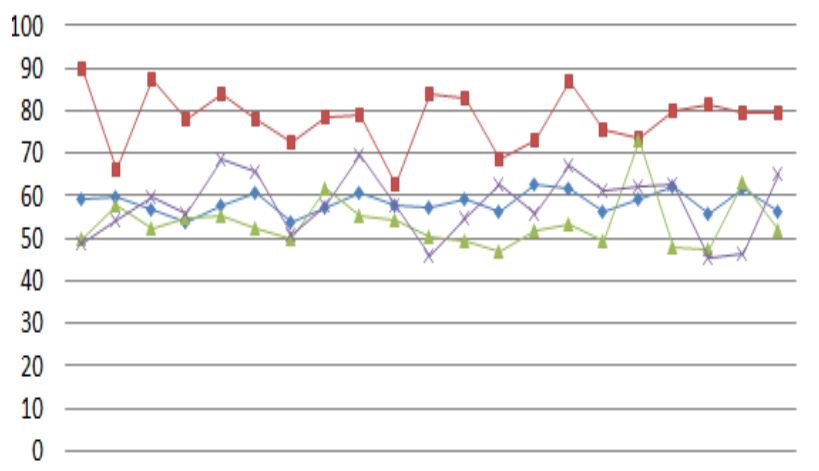

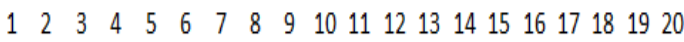

$$
\begin{aligned}
& \rightarrow \text { dataset } 1 \text {-dataset } ₫ \text {-dataset3 *dataset4 }
\end{aligned}
$$

Fig. 3. Classification accuracy with different value of parameters for four data set.

Table 1 shows the final result with the largest classification test with its parameter. The values are chosen from the result of Fig. 3. The dataset 1 get largest separation at parameter 14 as $62.78 \%$ of accuracy, while the dataset 2 largest at parameter 1 as $89.74 \%$. The dataset 3 largest at parameter 17 as $72.90 \%$ and the dataset 4 largest at parameter 9 as $69.83 \%$. Overall, this experiment results get the maximum value.
Table 1. The largest classification result with parameter along of each dataset.

\begin{tabular}{|c|c|c|}
\hline Dataset & Value of Parameters & Accuracy (\%) \\
\hline 1 & 14 & 62.78 \\
\hline 2 & 1 & 89.74 \\
\hline 3 & 17 & 72.90 \\
\hline 4 & 9 & 69.83 \\
\hline
\end{tabular}

\section{Conclusion}

To summarize, auditory stimuli could get the same sometime better than the visual stimuli. All the EEG signals recorded could be analyzed as visual stimuli to show the EEG signal of hand movement around the $\mu$ rhythms and more concentrate on channels C3 and C4. In the other hands, the noise data set to add data for iCSP method could reduce the invariant parts in the signals to get the higher separation in the classification. But it still based on the value of the adjusted parameter to have the fitness of the main data and added noise data. The result from table 2 show the recent study of stationary CSP method for 2 class separation. Overall, iCSP for auditory get the highest result, but the averaging of study [12] get the most stable method because of the invariant in the data set (the source of data).

In the future, we want to apply for more subject with various paradigm. The aim to make a BCI power system we will more concentrate to separate multi-class system in near future. We also will apply for BCI Competition data which supposed to recorded with the standard condition and then compare to original ICA algorithms. With BCI data for different kind of experiments and considered as a least noise data. In our case data, it is considered that the subject still not get much familiar with BCI systems so that also recorded the invariant and non stationary part in the EEG signals.

\section{References}

[1] W. Wojcikiewicz, C. Vidaurre, M. Kawanabe, "Stationary Common Spatial Patterns: Towards robust classification of non-stationary EEG signals," IEEE International Conference Acoustics, Speech and Signal Processing (ICASSP), pp. 577-580, 22-27 May 2011.

[2] F. V. Nijboer, A. Furdea, I. Gunst, J. Mellinger, D. J. McFarland, N. Birbaumer, A. Kübler, "An auditory brain-computer interface (BCI)”, Journal of Neuroscience Methods, vol. 167, no. 1, pp. 43-50, 2008

[3] G. Pfurtscheller and C. Neuper, "Motor imagery and direct brain-computer communication", 
Proceedings of the IEEE, vol. 89, no. 7, pp. 1123-1134. IEEE Press, Jul 2001.

[4] W. Samek, C. Vidaurre, K. R. Müller, M. Kawanabe,: "Stationary Common Spatial Patterns for Brain-Computer Interfacing," Journal of Neural Engineering, vol. 9, no. 2, pp. 026013 (pp14), 2012

[5] B. Blankertz, M. Kawanabe, R. Tomioka, F. U. Hohlefeld, V. Nikulin, and K.R. Müller,: "Invariant common spatial patterns: Alleviating nonstationarities in brain-computer interfacing," Advanced in Neural Information Processing Systems 20, vol. 20, pp. 113-120, 2008.

[6] Guger, C. Ramoser, G. Purftscheller. "Real-time EEG analysis with subject-specific spatial patterns for a brain-computer interface (BCI)," IEEE Transactions on Rehabilitation Engineering, vol. 8, no. 4, pp. 447-456, Dec 2000.

[7] M. G. Wentrup and M. Buss: "Multiclass common spatial patterns and information theoretic feature extraction," IEEE Transaction Biomedical Engineer, vol. 55, no. 8, pp. 1991-2000. 2008

[8] H. T. Nguyen, S. M. Park, K. E. Ko and K. B. Sim,: "Independent Component Analysis-based Robust Feature Extraction of Two Class-Hand Movements from Auditory Stimuli." in KIIS Spring Conference, vol. 22, no. 1, pp. 7-8, 2012

[9] E. Allwein, R. Schapire, and Y. Singer, "Reducing multiclass to binary: A unifying approach for margin classifiers," Journal of Machine Learning Research, vol. 1, pp. 113-141, 2000.

[10] T. H. Budzynski, H. K. budzynski, J. R. Evans and A. Abarbanel "Introduction of Quantitative EGG and Neurofeedback Second Edition Advanced Theory and Applications", Academic Press, chapter 6, pp. 143-166, 2009

[11] B. A. S. Hasan and J. Q. Gan, "Conditional random fields as classifiers for three-class motor-imagery brain-computer interfaces.," Journal of neural engineering, vol. 8, no. 2, pp. 025013, Apr. 2011.

[12] H. T. Nguyen, S. M. Park, K. E. Ko and K. B. Sim, "Invariant CSP-based binary classification method for hand movements analysis in EEG-based BCI system," KIIS Autumn Conference, vol. 22, no. 2, pp. 5-6, Nov 2012.

\section{저 자 소 개}

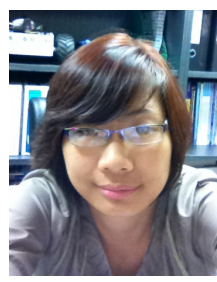

응웬 탄하(Thanh Ha Nguyen)

2010년 : Electrics Engineering, Hanoi University of Science Technology, Hanoi, Vietnam

2011년 현재 : Electronics and Electric Engineering, Chung ang University, Seoul, Republic of Korea.

관심분야 : Brain-Computer Interface, Machine Learning, Neuro-Robotics, Embedded System

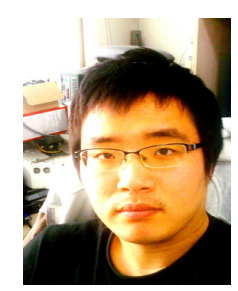

박승민(Seung-Min Park)

2010년 : 중앙대학교 전자전기공학부 공학사 2010년 현재 : 중앙대학교 대학원 전자전기공학부 석박사통합과정

관심분야 : Brain-Computer Interface, Intention Recognition Soft Computing

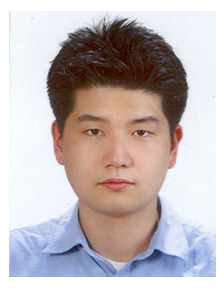

고광은(Kwang-Eun Ko)

2007년 : 중앙대학교 전자전기공학부 공학사 2007년 현재 : 중앙대학교 대학원 전자전기공학부 석박사통합과정

관심분야 : Multi-Agent Robotic Systems (MARS), Machine Learning, Context Awareness, Emotion Recognition Systems

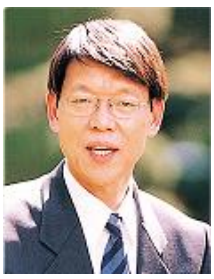

심귀보(Kwee-Bo Sim)

1990년 : The University of Tokyo 전자공학과 공학박사

1991년 현재 : 중앙대학교 전자전기공학부 교수 2006년 2007년 : 한국지능시스템학회 회장

관심분야 : 인공생명, 뇌-컴퓨터 인터페이스, 의도인식, 감성인 식, 유비쿼터스 지능형로봇, 지능시스템, 컴퓨테이 셔널 인텔리젼스, 지능형 홈 및 홈 네트워크, 유비쿼 터스 컴퓨팅 및 센서 네트워크, 소프트 컴퓨팅(신경 망, 퍼지, 진화연산), 다개체 및 자율분산로봇시스템, 인공면역시스템, 지능형 감시시스템 등.

Phone : +82-2-820-5319

E-mail :kbsim@cau.ac.kr

Homepage URL : http://alife.cau.ac.kr 